\title{
Long-term proton pump inhibitor use and the incidence of gastric cancer: A systematic review and meta-analysis
}

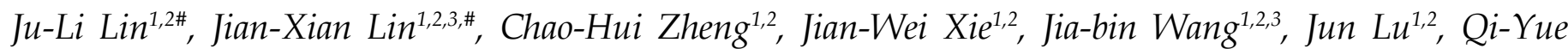
Chen $^{1,2}$, Long-long Cao ${ }^{1,2}$, Mi Lin ${ }^{1,2}$, Ping Li $i^{1,2,3^{*}}$, Chang-Ming Huang ${ }^{1,2,3^{*}}$

${ }^{1}$ Department of Gastric Surgery, Fujian Medical University Union Hospital, Fuzhou, Fujian Province, China.

${ }^{2}$ Department of General Surgery, Fujian Medical University Union Hospital, Fuzhou, Fujian Province, China.

${ }^{3}$ Key Laboratory of Ministry of Education of Gastrointestinal Cancer, Fujian Medical University, Fuzhou, Fujian Province, China.

\#Ju-Li Lin and Jian-Xian Lin contributed equally to this work and should be considered co-first authors.

\section{To Cite}

Lin J-L, Lin J-X, Zheng C-H, Xie J-W, Wang

J-B, Lu J, Chen Q-Y, Cao L-L, Lin M, Li

$\mathrm{P}$, Huang C-M, Long-term proton pump inhibitor use and the incidence of gastric cancer: A systematic review and metaanalysis. J Gastric Surg 2020; 2(1): 1-11

\section{Pubblication history}

Received: February 8, 2020

Accepted: February 24, 2020

Revised: February ,2020

Article impress: February 28, 2020

Published on line: March 15, 2020

\section{*Correspondence to}

Prof. Chang-Ming Huang,

Department of Gastric Surgery, Fujian

Medical University Union Hospital, No.29

Xinquan Road, Fuzhou 350001, Fujian

Province, China.

hcmlr2002@163.com;

Telephone: +86-591-83363366,

Fax: +86-591-83363366

\section{ABSTRACT \\ Background:}

There are controverted whether the long-term use of proton pump inhibitors (PPI) will increase the risk of gastric cancer. We performed a meta-analysis to assess the risk of gastric cancer in PPI users compared with non-PPI users.

\section{Methods:}

The main inclusion criteria were original studies reporting the incidence of gastric cancer in PPI users compared with non-PPI users. Key outcomes were the risk ratios (RR) for gastric cancer in association with PPI users or non-PPI users.

\section{Results:}

We analyzed data from 8 studies, comprising more than 927,684 patients. The risk of gastric cancer in PPI users was significantly higher than in non-PPI users $[R R=2.10$, 95\% CI (1.17-3.97)]. The risk of gastric cancer was similar between the 2 groups when the duration was $\leq 1$ year $[\mathrm{RR}=2.18,95 \% \mathrm{CI}(0.66-7.11)]$. While the risk of gastric cancer for PPI users was higher than in non-PPI users when the duration was between 1-3 years, $\geq 1$ year, $\geq 3$ years and $\geq 5$ years. The risk of non-cardiac gastric cancer for PPI users was higher than for non-PPI users $[\mathrm{RR}=2.66,95 \% \mathrm{CI}(1.66-4.27)]$, and the risk of non-cardiac gastric cancer for PPI users was higher than for non-PPI users when the duration $\geq 1$ year $[\mathrm{RR}=1.99,95 \% \mathrm{CI}(1.03-3.83)]$, but the risk for cardiac gastric cancer was similar between the 2 groups $[\mathrm{RR}=1.86,95 \% \mathrm{CI}(0.71-4.89)]$.

\section{Conclusions:}

We found the long-term use of PPI (duration $\geq 1$ year) was significantly associated with a higher risk of non-cardiac gastric cancer.

Key words:

proton pump inhibitors; gastric cancer; Helicobacter pylori infection; long-term use 


\section{Background:}

Since the introduction of the proton pump inhibitor (PPI) in the late 1980s[1], the outcomes of gastric acidrelated diseases have significantly improved. This innovation has significance in the treatment of gastric acid related diseases[2, 3]. Due to the outstanding efficacy and safety of PPIs, they have been widely used in clinical practice. The quantity of the prescriptions is increasing, and potential adverse effects have also attracted much attention. An increasing in the number of case reports and observational studies on the adverse events in patients receiving long-term PPI therapy had been reported. Currently, the most prominent concerns about long-term PPI use relate to the risks of bone fractures, enteric infection, pneumonia and vitamin B12 deficiency[4-7]. In recent years, studies[8] have shown that the long-term use of PPIs may increase the risk of gastric cancer, but these studies[9-11] are controversial. Helicobacter pylori ( $\mathrm{H}$. pylori) infection is one of the risk factors leading to gastric cancer, and PPIs are one of the major drugs used for the treatment of $\mathrm{H}$. pylori. The influence on the occurrence of gastric cancer needs further research. Therefore, the aim of this study is to assess the association between PPI use and risk of gastric cancer through systematic reviews and meta-analyses.

\section{Methods:}

All the search results were evaluated according to the Preferred Reporting Items for Systematic Reviews and Meta-Analyses (PRISMA) statement[12].

\section{Inclusion criteria and exclusion criteria}

The inclusion criteria are as follows: (1) RCTs or observational studies including cohort and case-control studies; (2) outcomes of PPI users were compared with those of non-PPI users; (3) studies provided adequate data that enabled the estimation of risk ratio (RR), odds ratio (OR), incidence rate ratio (IRR), and standardized incidence ratio (SIR). The exclusion criteria are as follows: (1) The article is a duplicate; (2) inadequate data; (3) sample size less than 20.

\section{Literature Search}

We conducted a comprehensive systematic literature search of online databases, including PubMed, the Cochrane Library, Embase and clinicaltrials.gov, from January 1, 1987 to Nov 1, 2018 to identify all RCTs and observational studies. The following key words were used in these literature searches: "proton pump inhibitor", "omeprazole", "esomeprazole", "pantoprazole", "lansoprazole", "dexlansoprazole", "rabeprazole", "gastric cancer", "gastric carcinoma", "gastric adenocarcinoma", "gastric neoplasm", gastric neoplasia", "stomach cancer", "stomach carcinoma", "stomach adenocarcinoma", "stomach neoplasm", and "stomach neoplasia". There were no language restrictions. We also reviewed the references of the included articles and related systematic reviews to identify additional studies.

\section{Study Selection and Quality Assessment}

The quality of included non-RCTs was assessed using the Newcastle-Ottawa Scale (NOS) [13]. The scale used a score system, which ranged from 0 to 9 , and the quality of the observational studies were enrolled if they achieved 6 or more.

\section{Data Extraction}

Data extraction and the evaluation of literature quality were conducted independently by 2 investigators (Ju-li Lin and Jian-xian Lin). When there was any uncertainty about the inclusion of a study, the issue was discussed between the two investigators to achieve a resolution. In cases of disagreement, the qualitative analysis was performed by Chao-Hui Zheng. A Microsoft Excel database was used to record all available information, including baseline details, title, first author's name, year of publication, study design, region, journal, sample size, period of patient recruitment, follow-up time, definition of PPI use, adjusted odds ratio (OR), risk ratio (RR), standardized incidence ratio (SIR), and incidence rate ratio (IRR) of gastric cancer.

\section{Outcome definition}

Primary outcome: the risk of gastric cancer in PPI users compared with non-PPI users; Secondary outcomes: the risk of gastric cancer when therapy duration $\leq 1$ year, 1-3 years, $\geq 1$ year, $\geq 3$ years and $\geq 5$ years; the risk of cardia gastric cancer; the risk of non-cardia gastric cancer; the risk of gastric cancer with $\mathrm{H}$. pylori infection; and the risk of gastric cancer with prior $\mathrm{H}$. pylori infection.

\section{Data synthesis}

Because the absolute risk of gastric cancer is low, one can generally ignore the distinctions among the various measures of relative risk (e.g., odds ratio, risk ratio, standardized incidence ratio, incidence rate ratio)[14, 15]. The effect estimates that were extracted, if available, or de novo calculated from available data were SIR, IRR, RR and OR. SIR was estimated as the ratio of the observed over expected number of cases for exposed patients. The 95\% confidence interval (CI) for loge(SIR) was constructed via the term" \pm " 1.96/ [square $\operatorname{root}(\mathrm{O})$ ], where $\mathrm{O}$ was the observed number of events (Alder et al, 2006)[16]. Maximally adjusted effect estimates (ORs) were additionally extracted on the total of the sample, wherever possible.

\section{Statistical Analysis}

The pooled risk ratio (RR) with 95\% confidence intervals (95\% CIs) was estimated for dichotomous outcomes. Single-arm meta-analyses were performed for the PPI and non-PPI groups. Cumulative metaanalyses were also performed to evaluate the stability of the effect sizes. The Cochran's Q statistic and the I2 statistics were used to assess the heterogeneity among all studies. Heterogeneity among studies was tested using Cochran's Chi-square test and I2, in which I2 > $50 \%$ suggested significant heterogeneity. A randomeffects model was chosen to pool the results when I2 $>50 \%$, while a fixed-effects model was used when $\mathrm{I} 2<$ $50 \%$. When possible, subgroup analyses were performed to assess the potential impact of the duration of PPI exposure, tumor location and $\mathrm{H}$. pylori infection. $\mathrm{P}<$ 
0.05 was considered to represent statistical significance (2-sided). All the statistical analyses were conducted using STATA, version 13.0 (Stata Corporation, College Station, TX).

\section{Results:}

\section{Studies Retrieved and Characteristics}

According to the previous search strategy, 977 citations were obtained from the online database from January 1, 1987 to May 1, 2018. A total of 960 articles were excluded by viewing the titles and abstracts. The full texts of 17 records were read. Among the remaining 17 records, 8 letter and 1 case-control study were removed (supplement reference). Finally, 8 full-text studies were obtained and assessed according to the eligibility criteria, including 4 case-control studies and 4 cohort studies, comprising more than 927,684 patients. The detailed literature search and screening process are shown in Figure 1. The characteristics included in the study are shown in Table 1, including the first author's name, year of publication, study design, region, journal, sample size, period of patient recruitment patients, follow-up time and definition of PPI use.

The quality of 8 studies was assessed using the Newcastle-Ottawa Scale (NOS). Two studies achieved a NOS score of 6 , three studies achieved a NOS score of 7 and three studies achieved a NOS score of 8 (Table 2). Six studies had a clear follow-up time, and four studies had a median follow-up period $>3$ years. The longest median follow-up period was 7.6 years. Six studies had a clear definition of the use of PPIs. Seven studies compared the risk of gastric cancer between PPI users and non-PPI users.

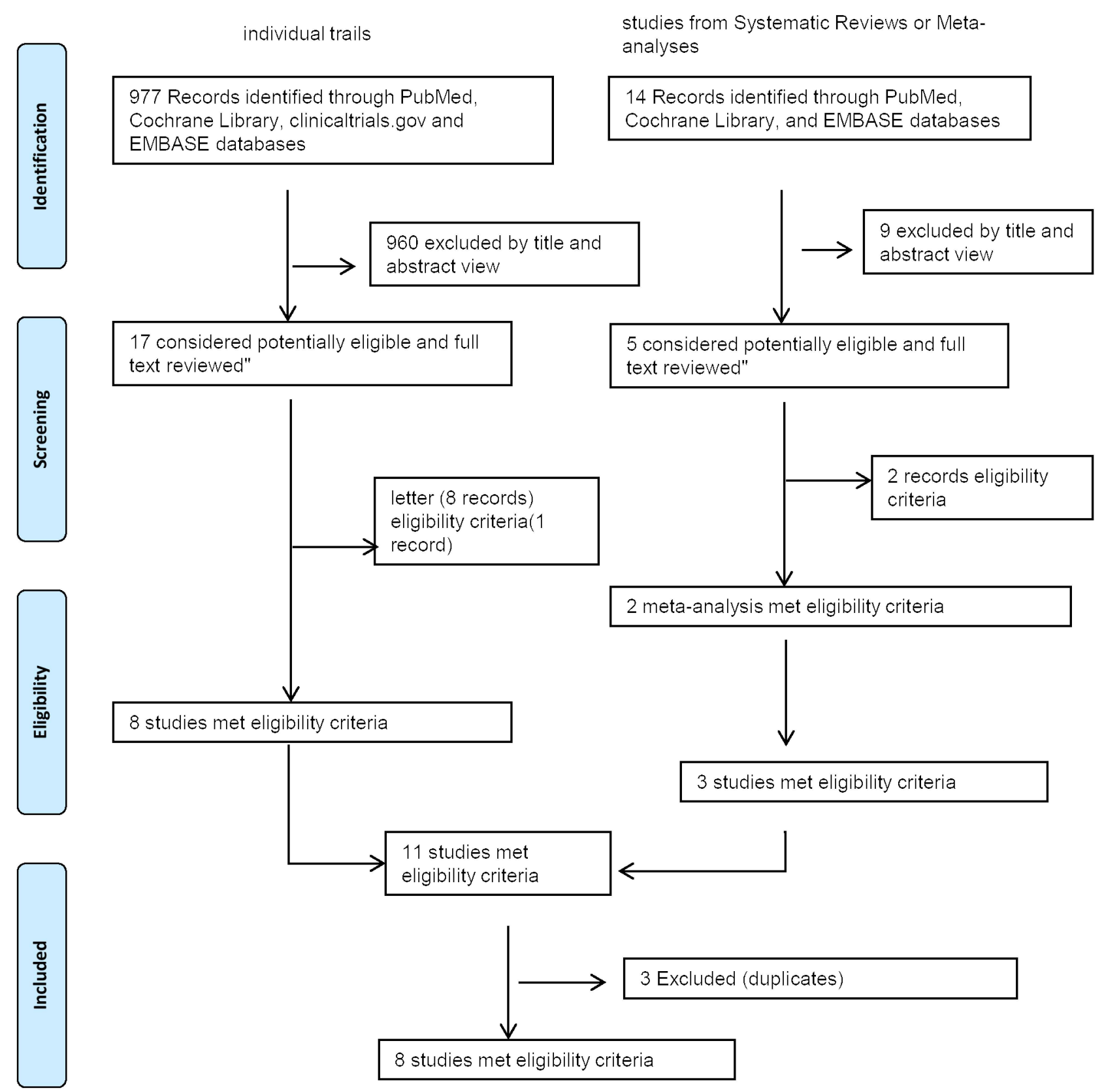

Figure 1: Literature Search and Screening Process. 


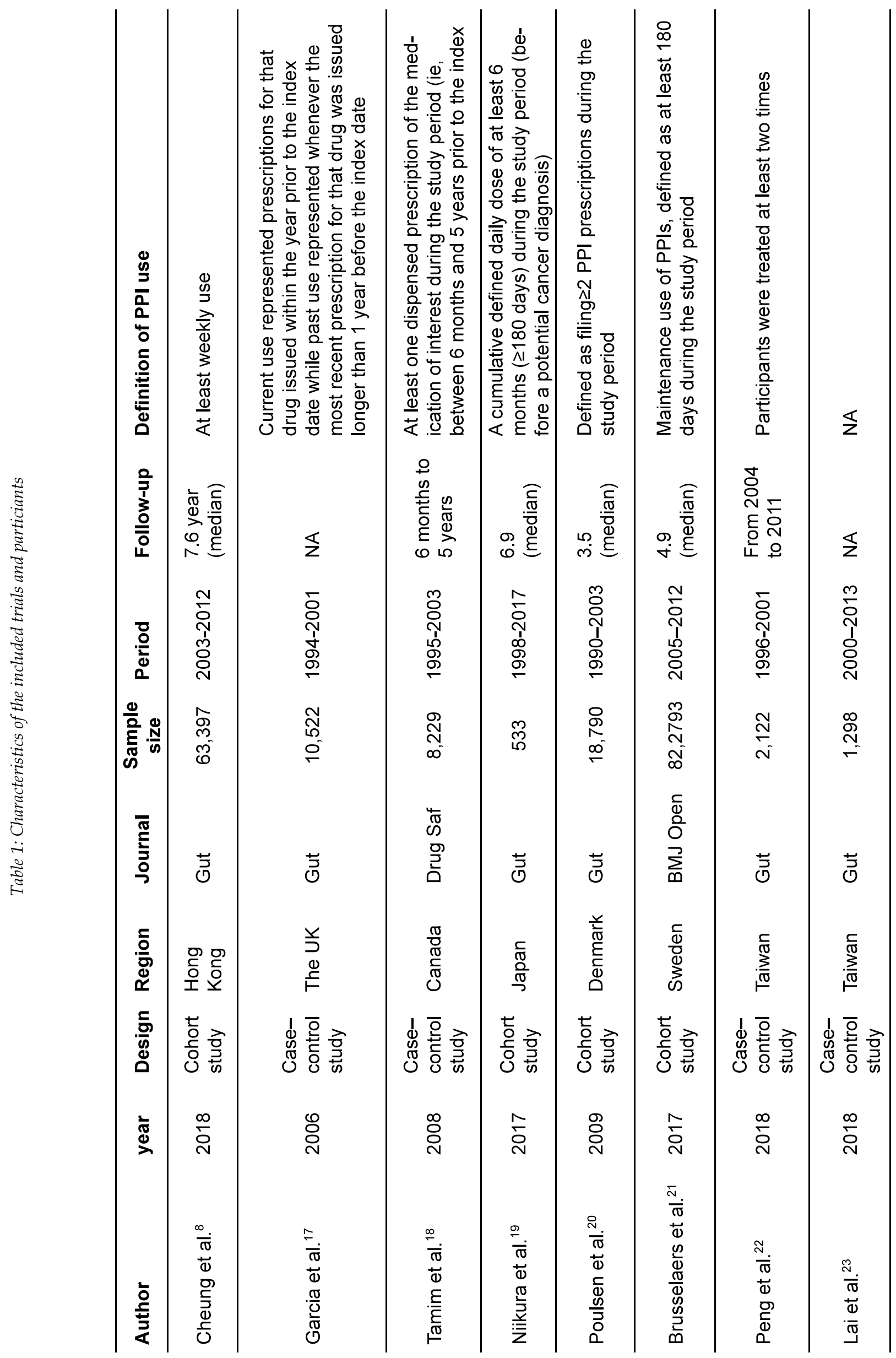



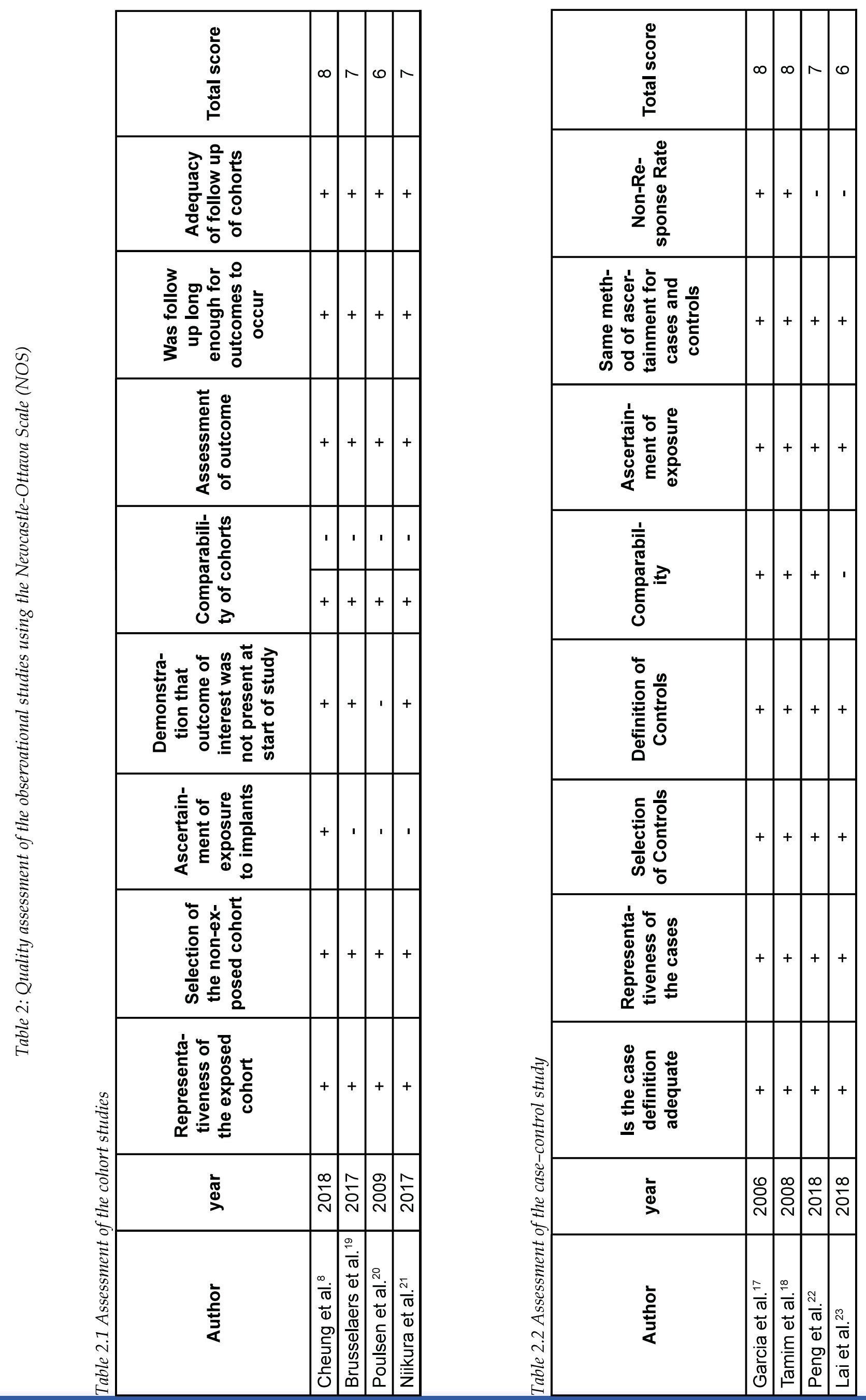
The risk of gastric cancer in PPI users compared with nonPPI users

Seven studies involving 926,386 patients compared the risk of gastric cancer in PPI users compared with non-PPI users: Cheung et al[8], Garcia et al[17], Tamim et al[18], Niikura et al[19], Poulsen et al[20], Brusselaers et al[21], and Peng YC et al[22]. As shown in Figure 2A, the risk of gastric cancer PPI users was significantly higher than non-PPI users [RR=2.10, 95\% CI (1.17-3.97)]. Regional variations are also analyzed low-intermediate incidence vs high incidence. Cheung et al[8], Niikura et al[19] and Peng YC et al[22] is from high incidence region(HK, Japan,
Taiwan). Garcia et al[17], Tamim et al[18], Poulsen et al[20] and Brusselaers et al[21] is from low-intermediate incidence region (UK, Canada, Denmark, Sweden). We found the risk of gastric cancer PPI users was significantly higher than non-PPI users [RR=2.53, 95\% CI (2.03-3.17)] in high incidence region, but no significant differences were seen between the two groups [RR=1.66, 95\% CI (0.95-2.89)] in low incidence region. The result of cumulative metaanalysis showed that the significant difference supporting PPI users was first found in the latest study in 2008, with the $\mathrm{CI}$ narrowing and the effect size becoming stable (Figure 2B).
2A

study
$\%$

$\operatorname{RR}(95 \% \mathrm{Cl}) \quad$ Weight

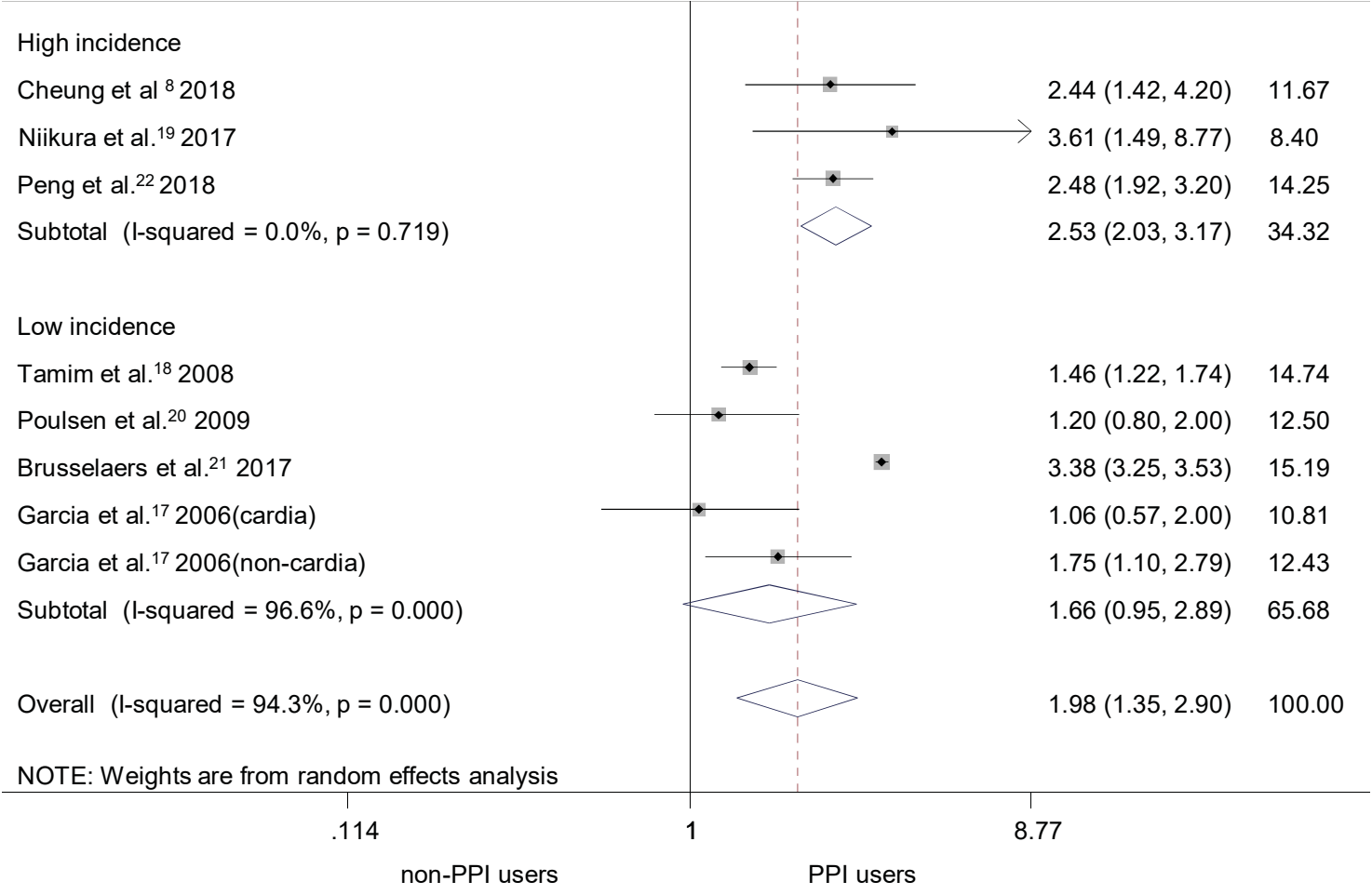

Figure 2A: Forest plot of pooled risk ratio for gastric cancer in PPI users versus non-PPI users.

2B

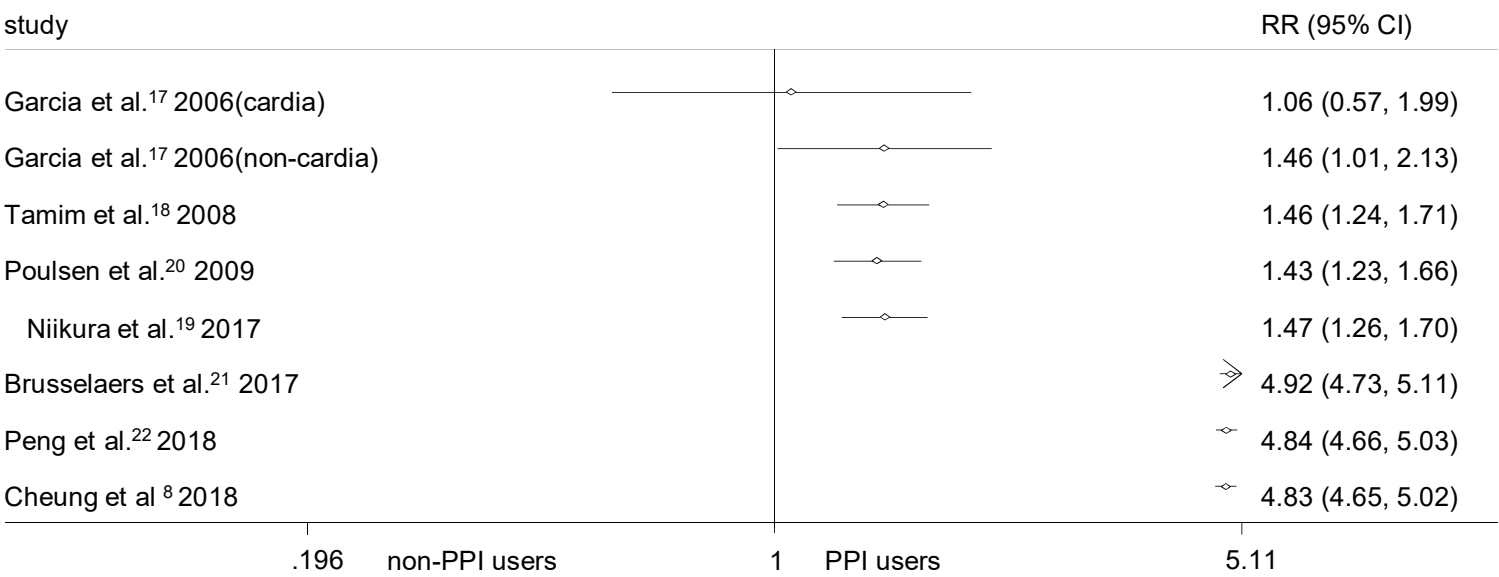

Figure 2B: Cumulative meta-analysis of the risk ratio for the gastric cancer according to time. 


\section{Subgroup analysis according to duration}

Duration $\leq 1$ year: Five studies enrolled 15,494 patients including Garcia et al[17], Poulsen et al[20], Brusselaers et al[21], and Lai et al[23]. No significant differences were seen between the two groups [RR=2.18, 95\% CI (0.66-7.11)] (Figure 3).

Duration 1-3 years: Two studies enrolled 12,715 patients, including Garcia et al[17] and Brusselaers et al[21]. The risk of gastric cancer PPI users was significantly higher than non-PPI users [RR=1.74, 95\% CI (1.04-2.90)] (Figure $3)$.

Duration $\geq 1$ year: Four studies enrolled 93,807 patients, including Cheung et al[8], Garcia et al[17], Poulsen et al[20], and Brusselaers et al[21]. The risk of gastric cancer PPI users was significantly higher than in non-PPI users
[RR=1.88, 95\% CI (1.60-2.22),] (Figure 3).

Duration $\geq 3$ years: Four studies enrolled 93,807 patients, including Garcia et al[17], Poulsen et al[20], and Brusselaers et al[21]. The risk of gastric cancer PPI users was significantly higher than non-PPI users $[R R=1.95,95 \%$ CI (1.65-2.31)] (Figure 3).

Duration $\geq 5$ years: Four studies enrolled 19,323 patients, including Poulsen et al[20] and Brusselaers et al[21]. The risk of gastric cancer in PPI users was significantly higher than in non-PPI users [RR=2.03, 95\% CI (1.75-2.35)] (Figure 3).

Furthermore, the risk increased with a longer duration of PPI use (RR=1.74, 95\% CI (1.04-2.90) for 1-3 years of use; $R R=1.95,95 \%$ CI (1.65-2.31) for $\geq 3$ years of use and $R R=2.03,95 \%$ CI (1.75-2.35) for $\geq 5$ years of use).

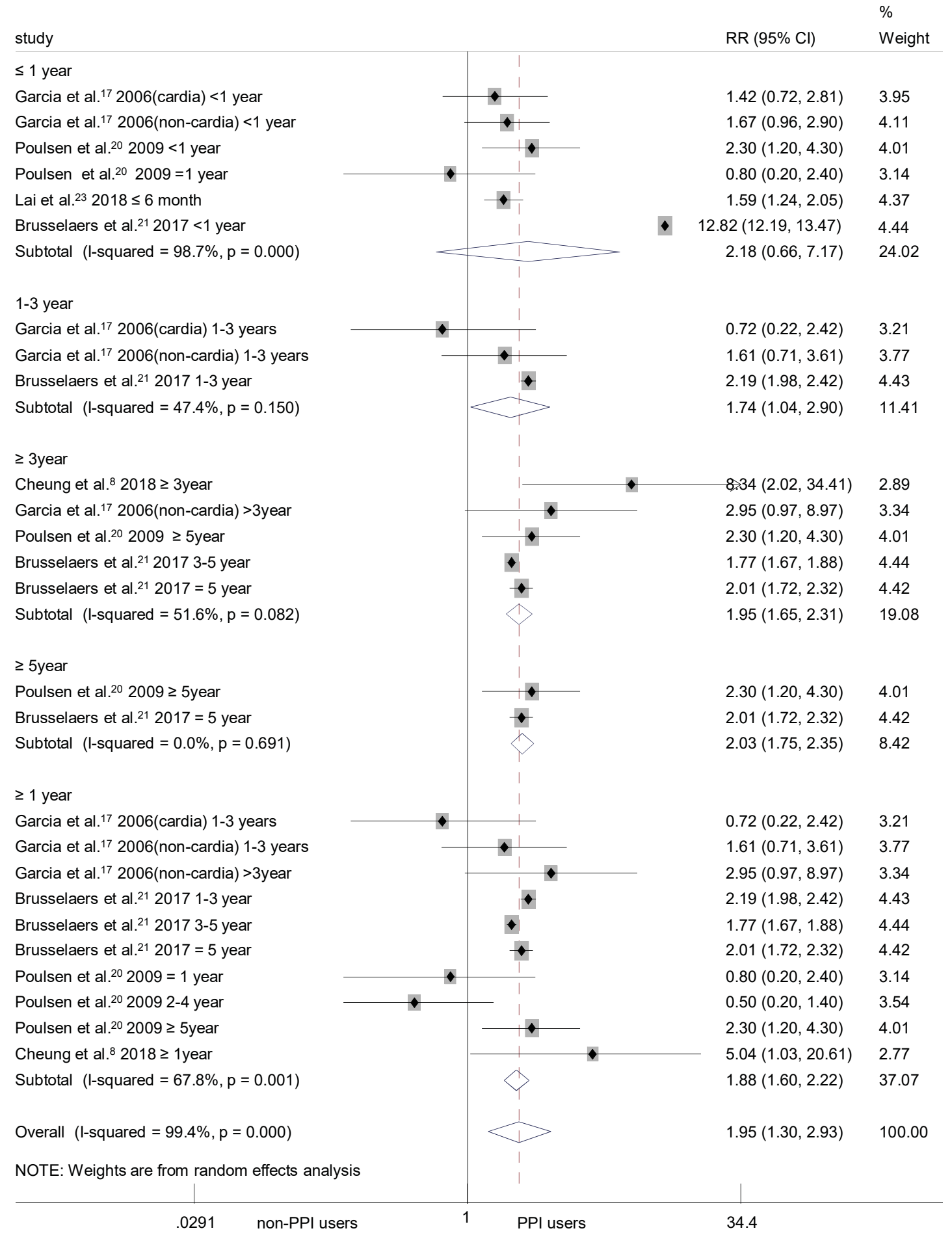

Figure 3: Forest plot of the pooled risk ratio for gastric cancer in PPI users versus non-PPI users according to duration. 


\section{Subgroup analysis according to location}

Four studies enrolled 12,294 patients, including Cheung et al[8], Garcia et al[17], Tamim et al[18], Brusselaers et al[21], and Peng YC et al[22]. The risk of non-cardia gastric cancer in PPI users was significantly higher than in non-PPI users $[R R=2.66,95 \%$ CI (1.66 -4.27)]. However, no significant differences were found between the two groups for the risk of cardia gastric cancer [OR=1.86, 95\% CI (0.71-4.89)]
(Figure 4A).

In Garcia et al[17], the risk of non-cardiac gastric cancer for PPI users was higher than non-PPI users when the duration $\geq 1$ year $[R R=1.99,95 \%$ CI (1.03 -3.83)] (Figure 4B). Cheung et al[8], Tamim et al[18], Brusselaers et al[21] and Peng YC et al[22] included only gastric cancer, while Garcia et al[17] included only gastric adenocarcinoma.

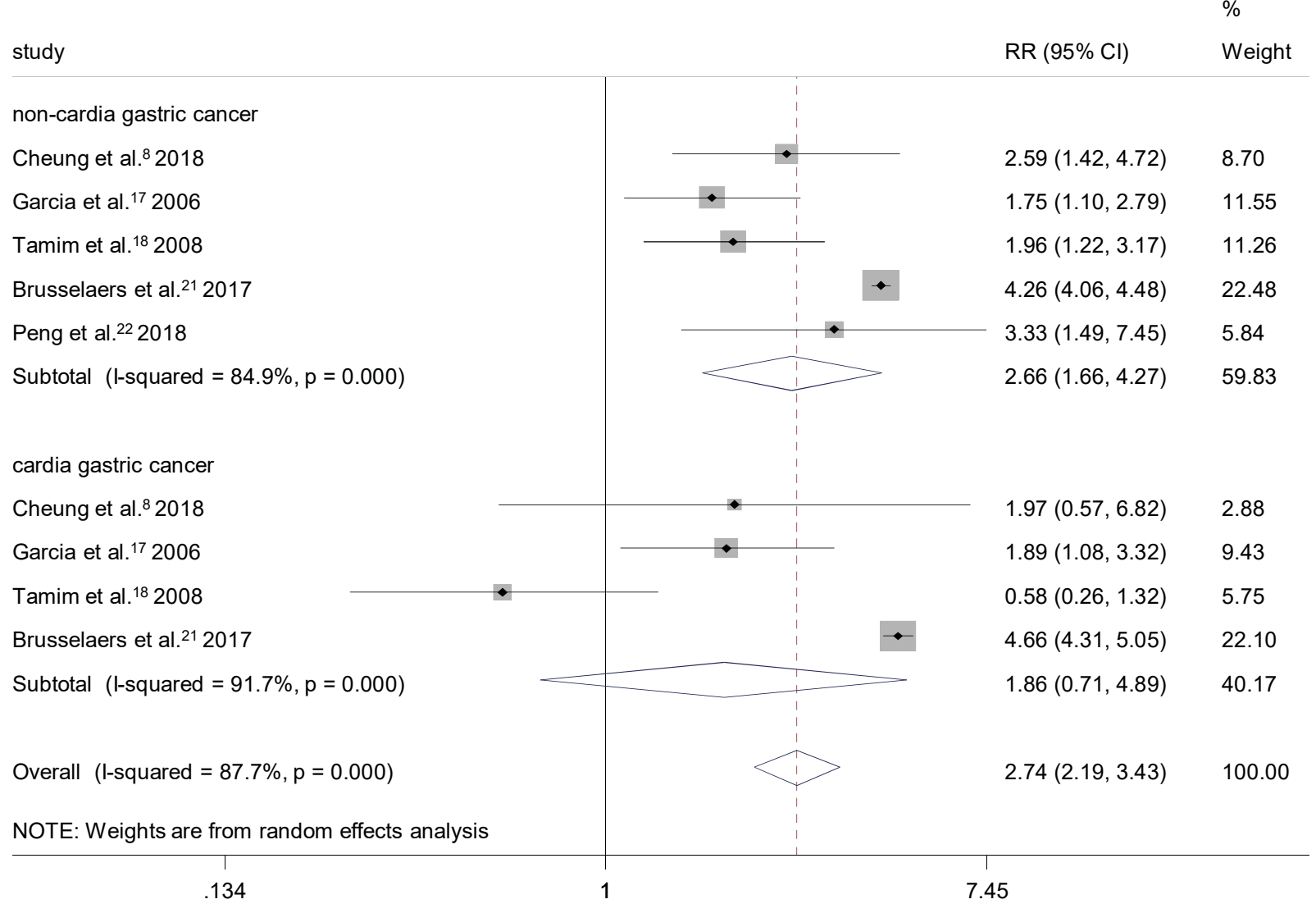

Figure 4A: Forest plot of the pooled risk ratio for gastric cancer in PPI users versus non-PPI users according to location.

$\%$

study

$\operatorname{RR}(95 \% \mathrm{Cl})$

Weight

Garcia et al. ${ }^{17} 2006$ (non-cardia) $1-3$ years

Garcia et al. ${ }^{17} 2006$ (non-cardia) $\geq 3$ year

Overall $(\mathrm{I}$-squared $=0.0 \%, \mathrm{p}=0.389$ )

NOTE: Weights are from random effects analysis
$2.95(0.97,8.97)$

$1.61(0.71,3.61)$

$1.99(1.03,3.83)$

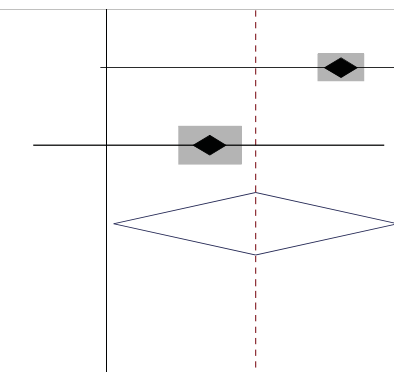

PPI users

Figure 4B: Forest plot of the pooled risk ratio for non-cardia gastric cancer in PPI users versus non-PPI users when duration $\geq 1$ year. 


\section{Subgroup analysis according to H. pylori infection}

Three studies enrolled 845,923 patients, including Cheung et al[8], Niikura et al[19], and Brusselaers et al[21]. The risk of gastric cancer in PPI users was significantly higher than in non-PPI users with prior $H$. pylori infection $[R R=4.8$, 95\% CI (1.82 -12.67)] (Figure 4C).

Three studies enrolled 123,915 patients, including Cheung et al[8], Brusselaers et al[21], and Lai et al[23]. The risk of gastric cancer was similar between the two groups with $\mathrm{H}$. pylori infection $[\mathrm{OR}=0.91,95 \% \mathrm{CI}(0.17-4.90)]$ (Figure $4 c$ ). Cheung et al[8] included 142460 PPIs users without prior $\mathrm{H}$. pylori eradication therapy were identified with a total of 705094 person-years of follow-up. Niikura et al[19] included 571 patients who achieved H.pylori eradication were selected using thedatabase of University Tokyo Hospital from 1998 to 2017.

\section{Discussion:}

This study included recent studies with large sample sizes from 1987 to 2018 to explore the risk of gastric cancer in PPI users compared with non-PPI users. Although all the included studies were retrospective studies, they were of were relatively high quality according to the results of quality evaluation and had large sample sizes. Our study included 8 publications with 926,386 patients, and the data suggested that long-term PPI use increases the risk of gastric cancer. Subgroup analysis suggested that longterm PPI use may increase the risk of non-cardiac gastric cancer when the duration is $\geq 1$ year. Long-term PPI use after $\mathrm{H}$. pylori eradication therapy for patients with prior $\mathrm{H}$. pylori infection may increase the risk of gastric cancer. In recent years, the reported incidence rates of gastric cancer in PPI patients has been $0.081 \%-5.0 \%[8,17,20$, 21]. This rate is significantly higher than the incidence of gastric cancer in the general population $(0.014 \%-0.491 \%)$ $[24,25]$. In our study, the risk of gastric cancer in PPI users was also significantly higher than that in non-
PPI users. Regional variations also be analyzed (lowintermediate incidence (Sweden, Denmark, Canada, UK) vs high incidence (Taiwan, Japan, HK)). Subgroup analysis according to incidence, the risk of gastric cancer PPI users was significantly higher than non-PPI users $[R R=2.53,95 \%$ CI (2.03-3.17)] in high incidence region, but no significant differences were seen between the two groups [RR=1.66, 95\% CI (0.95-2.89)] in low incidence region. Currently, the mechanism by which a PPI may increase the occurrence of gastric cancer has not been fully elucidated. Some studies suggest that the long-term use of PPIs profoundly reduces gastric acid production and consequently leads to the increased secretion of gastrin. Hypergastrinemia as a result of acid suppression causes the hyperplasia of enterochromaffin-like cells, resulting in the formation of microcarcinomas and gastric neuroendocrine tumors[26-29]. Song et al suggest that a PPI inhibits gastric acid and leads to hypergastrinemia, which may lead to hyperproliferation, chronic hypochloremia, chronic inflammation, intestinal metaplasia and atrophy of the stomach [30]. In addition, a high $\mathrm{pH}$ environment can cause double infection with $\mathrm{H}$. pylori and non-H. pylori bacterial species[31, 32].The synergistic effect of many bacteria can produce nitrosamine carcinogens, which may lead to the development of gastric cancer.

However, does the correlation between PPI use and gastric cancer depend on the time of treatment? Cheung et al [8] thought that PPIs increase the risk of gastric cancer development in the context of underlying $\mathrm{H}$. pyloriassociated chronic gastritis and atrophy. A meta-analysis [33] revealed that the long-term use of PPIs ( $\geq 12$ months) is associated with an increased risk of fundic gland polyps. Suissa $S$ et al [10] thought that the correlation between the use of PPIs and the risk of gastric cancer may be caused by time bias. In this study, we found that there was a significant time correlation between a PPI and the incidence of gastric cancer. The incidence of gastric cancer

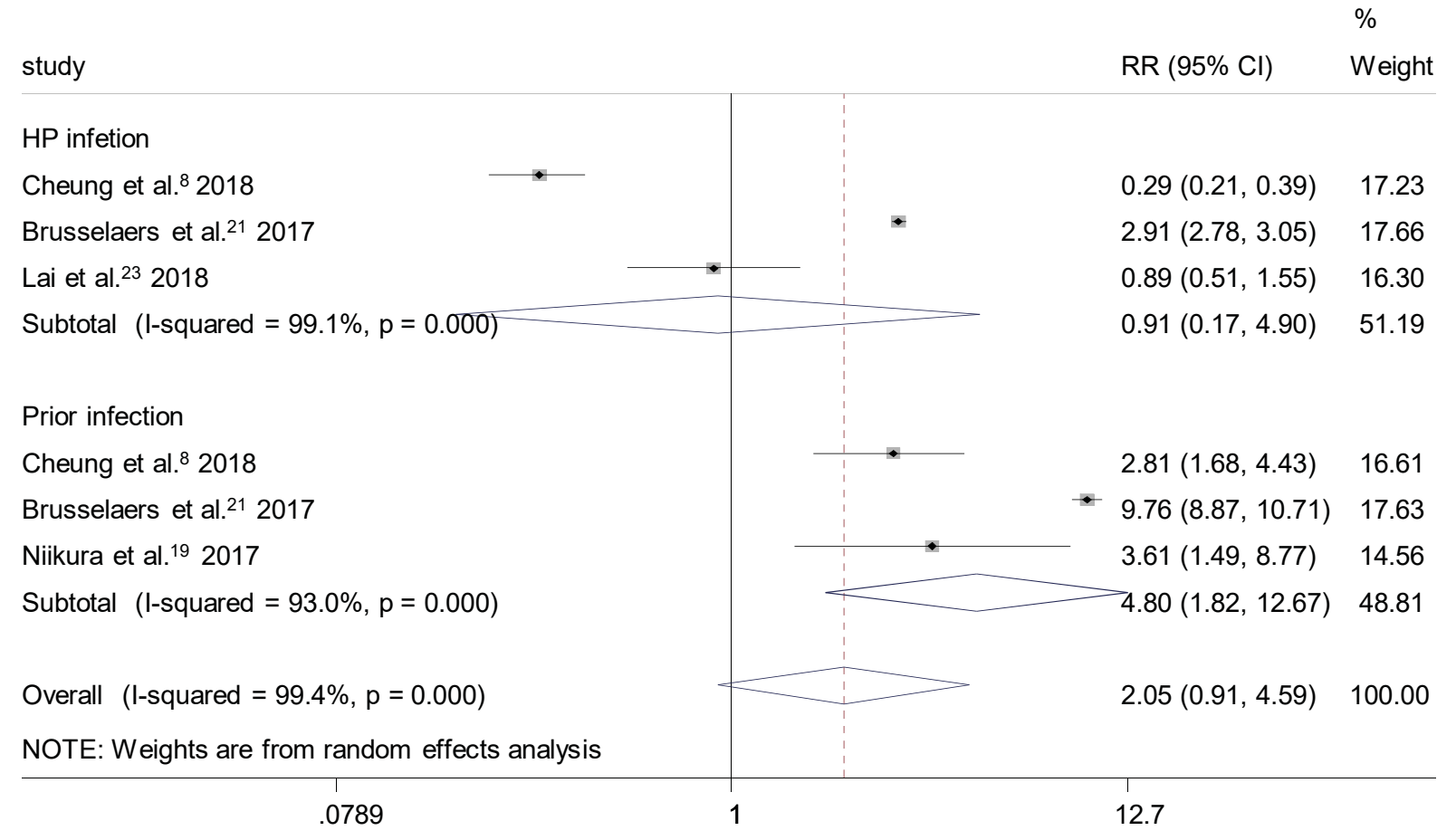

Figure 4C: Forest plot of pooled risk ratio for gastric cancer in PPI users versus non-PPI users according to H. pylori infection. 
was significantly higher when a PPI was used for $\geq 1$ year. Because of the different functional cells on different parts of stomach, the study found that long-term PPI use may increase the incidence rate of non-cardia cancer according to stratification analysis of location. This may be because gastrin is secreted by $G$ cells in the mucosa of the gastric antrum and proximal duodenum. These locations tolerate gastric acid well but are more prone to intestinal metaplasia and precancerous lesions after gastric acid suppression. In addition, PPIs are one of the main drugs for the treatment of H. pylori[4], but they are often abused. Subgroup analysis found that the risk of gastric cancer in PPI users was significantly higher than non-PPI users with prior $\mathrm{H}$. pylori infection, while there was no statistically significant difference between PPI users and non-PPI users with $\mathrm{H}$. pylori infection. However, while is relatively safe after H. pylori eradication, it is not appropriate to prescribe long-term PPIs to these patients, even after successful eradication of $\mathrm{H}$. pylori.

There are also some limitations in this study. First, there is certain publication bias and selection bias because of the retrospective design of the original research. Second, the inconsistencies in the definition, dosage, duration, type of PPI use and the inclusion criteria of the original study may lead to bias. In addition, other potential confounding factors include precancerous diseases of gastric cancer, such as gastric polyps, gastroesophageal reflux disease and peptic ulcers, and a family history of gastric cancer. Those factors have not been systematically evaluated because of the lack of related data. However, based on the results of meta-analysis, this study shows that the long-term use of a PPI was associated with an increased risk of gastric cancer development, particularly for non-cardia cancer and in high incidence region, which is of great significance for the rational clinical application of PPIs.

\section{Acknowlwdgements \\ Not applicable}

\section{Contributors}

$\mathrm{CMH}$, JXL and JLL conceptualized and designed the study, acquired and analysed data, interpreted the study results, drafted the manuscript and critically revised the manuscript for important intellectual content. $\mathrm{CHZ}$ and PL acquired and analysed data, interpreted the study results and critically revised the manuscript for important intellectual content. JWX and JBW designed the study, interpreted the study results and critically revised the manuscript for important intellectual content. JL and QY designed the study, interpreted the study results and critically revised the manuscript for important intellectual content. LLC and ML conceptualized and designed the study, interpreted the study results and critically revised the manuscript for important intellectual content.

\section{Funding}

Scientific and technological innovation joint capital projects of Fujian Province, China (No.2016Y9031). Construction Project of Fujian Province Minimally Invasive Medical Center (No. [2017]171). Fujian province medical innovation project (2015-CXB-16).

\section{Competing interests}

No benefits in any form have been received or will be received from a commercial party related directly or indirectly to the subject of this article.

\section{Availability of data and materials}

Further information are available from the corresponding author on reasonable request.

\section{Ethics approval}

Our protocol was approved by the ethics committee of the Fujian Medical University Union Hospital.

All procedures followed were in accordance with the ethical standards of the responsible committee on human experimentation (institutional and national) and with the Helsinki Declaration of 1964 and later versions. Informed consent or substitute for it was obtained from all patients for being included in the study.

\section{Provenance and peer review}

Not commissioned; externally peer reviewed.

\section{Open access}

This is an Open Access article distributed in accordance with the Creative Commons Attribution Non-Commercial (CC BY-NC 4.0) license, which permits others to distribute, remix, adapt, build upon this work noncommercially, and license their derivative works on different terms, provided the original work is properly cited and the use is noncommercial. See: http:/ / creativecommons.org/licenses/ by-nc/4.0/

\section{References}

[1] Shin JM, Sachs G. Pharmacology of proton pump inhibitors. Current gastroenterology reports. 2008;10:528-34.

[2] Liu WZ, Xie Y, Lu H, Cheng H, Zeng ZR, Zhou LY, et al. Fifth Chinese National Consensus Report on the management of Helicobacter pylori infection. Helicobacter. 2018;23:e12475.

[3] Malfertheiner P, Megraud F, O'Morain CA, Gisbert JP, Kuipers EJ, Axon AT, et al. Management of Helicobacter pylori infection-the Maastricht V/Florence Consensus Report. Gut. 2017;66:6-30.

[4] Vaezi MF, Yang YX, Howden CW. Complications of Proton Pump Inhibitor Therapy. Gastroenterology. 2017;153:35-48.

[5] Voelker R. Proton pump inhibitors linked to fracture risk. Jama. 2010;304:29.

[6] Littman A. Potent acid reduction and risk of enteric infection. Lancet. 1990;335:222.

[7] Lam JR, Schneider JL, Zhao W, Corley DA. Proton pump inhibitor and histamine 2 receptor antagonist use and vitamin B12 deficiency. Jama. 2013;310:2435-42.

[8] Cheung KS, Chan EW, Wong AYS, Chen L, Wong ICK, Leung WK. Long-term proton pump inhibitors and risk of gastric cancer development after treatment for Helicobacter pylori: a population-based study. Gut. 2018;67:28-35.

[9] Gueta I, Halkin H, Markovits N, Loebstein R. Proton pump inhibitors and the risk for gastric cancer: possible confounding by serum vitamin B 12. Gut. 2017.

[10] Suissa S, Suissa A. Proton-pump inhibitors and increased gastric cancer risk: time-related biases. Gut. 2018.

[11] Suzuki H, Matsuzaki J. Helicobacter pylori eradication failure may have confounded the recent large-scale health database study that showed proton pump inhibitors increase gastric cancer risk. Gut. 2017.

[12] Moher D, Liberati A, Tetzlaff J, Altman DG, Group P. Preferred reporting items for systematic reviews and meta-analyses: the PRISMA statement. Bmj. 2009;339:b2535.

[13] Stang A. Critical evaluation of the Newcastle-Ottawa scale for the assessment of the quality of nonrandomized studies in meta-analyses. European journal of epidemiology. 2010;25:603-5.

[14] Greenland S. Quantitative methods in the review of epidemiologic literature. Epidemiologic reviews. 1987;9:1-30.

[15] Siristatidis C, Sergentanis TN, Kanavidis P, Trivella M, Sotiraki M, Mavromatis I, et al. Controlled ovarian hyperstimulation for IVF: impact 
on ovarian, endometrial and cervical cancer--a systematic review and meta-analysis. Human reproduction update. 2013;19:105-23.

[16] Alder N, Fenty J, Warren F, Sutton AJ, Rushton L, Jones DR, et al. Meta-analysis of mortality and cancer incidence among workers in the synthetic rubber-producing industry. American journal of epidemiology. 2006;164:405-20

[17] Garcia Rodriguez LA, Lagergren J, Lindblad M. Gastric acid suppression and risk of oesophageal and gastric adenocarcinoma: a nested case control study in the UK. Gut. 2006;55:1538-44.

[18] Tamim H, Duranceau A, Chen LQ, Lelorier J. Association between use of acid-suppressive drugs and risk of gastric cancer. A nested casecontrol study. Drug safety. 2008;31:675-84

[19] Niikura R, Hayakawa Y, Hirata Y, Yamada A, Fujishiro M, Koike $\mathrm{K}$. Long-term proton pump inhibitor use is a risk factor of gastric cancer after treatment for Helicobacter pylori: a retrospective cohort analysis. Gut. 2017.

[20] Poulsen AH, Christensen S, McLaughlin JK, Thomsen RW, Sorensen HT, Olsen JH, et al. Proton pump inhibitors and risk of gastric cancer: a population-based cohort study. British journal of cancer. 2009;100:1503-7. [21] Brusselaers N, Wahlin K, Engstrand L, Lagergren J. Maintenance therapy with proton pump inhibitors and risk of gastric cancer: a nationwide population-based cohort study in Sweden. BMJ open 2017;7:e017739.

[22] Peng YC, Huang LR, Lin CL, Hsu WY, Chang CS, Yeh HZ, et al. Association between proton pump inhibitors use and risk of gastric cancer in patients with GERD. Gut. 2018

[23] Lai SW, Lai HC, Lin CL, Liao KF. Proton pump inhibitors and risk of gastric cancer in a case-control study. Gut. 2018.

[24] Chen W, Zheng R, Baade PD, Zhang S, Zeng H, Bray F, et al. Cancer statistics in China, 2015. CA: a cancer journal for clinicians. 2016;66:11532

[25] Siegel RL, Miller KD, Jemal A. Cancer statistics, 2018. CA: a cancer journal for clinicians. 2018;68:7-30.

[26] Waldum HL, Aase S, Kvetnoi I, Brenna E, Sandvik AK, Syversen $\mathrm{U}$, et al. Neuroendocrine differentiation in human gastric carcinoma. Cancer. 1998;83:435-44

[27] Havu N. Enterochromaffin-like cell carcinoids of gastric mucosa in rats after life-long inhibition of gastric secretion. Digestion. 1986;35 Supp 1:42-55.

[28] Poynter D, Pick CR, Harcourt RA, Selway SA, Ainge G, Harman IW, et al. Association of long lasting unsurmountable histamine $\mathrm{H} 2$ blockade and gastric carcinoid tumours in the rat. Gut. 1985;26:1284-95.

[29] Bakkelund K, Fossmark R, Nordrum I, Waldum H. Signet ring cells in gastric carcinomas are derived from neuroendocrine cells. The journal of histochemistry and cytochemistry : official journal of the Histochemistry Society. 2006;54:615-21.

[30] Song H, Zhu J, Lu D. Long-term proton pump inhibitor (PPI) use and the development of gastric pre-malignant lesions. The Cochrane database of systematic reviews. 2014:CD010623.

[31] Ahn JS, Eom CS, Jeon CY, Park SM. Acid suppressive drugs and gastric cancer: a meta-analysis of observational studies. World journal of gastroenterology. 2013;19:2560-8.

[32] Engstrand L, Lindberg M. Helicobacter pylori and the gastric microbiota. Best practice \& research Clinical gastroenterology. 2013;27:3945.

[33] Tran-Duy A, Spaetgens B, Hoes AW, de Wit NJ, Stehouwer CD. Use of Proton Pump Inhibitors and Risks of Fundic Gland Polyps and Gastric Cancer: Systematic Review and Meta-analysis. Clinical gastroenterology and hepatology : the official clinical practice journal of the American Gastroenterological Association. 2016;14:1706-19 e5. 